\title{
ANH
}

\section{Conhecimento de pacientes sobre anticoagulação oral após o implante de valva metálica}

\author{
Carolina Galli da Silveira1, Paolla Algarte Fernandes², Suellen Rodrigues de Oliveira Maier ${ }^{3}$, Carina \\ Aparecida Marosti Dessotte 4
}

\section{RESUMO}

Objetivo: avaliar o conhecimento sobre anticoagulação oral de pacientes após a primeira cirurgia cardíaca com implante de valva metálica. Método: estudo descritivo de corte transversal e abordagem quantitativa, no qual foram entrevistados 32 pacientes, com idade superior a 18 anos, submetidos a implante de prótese cardíaca metálica em um hospital de ensino. A coleta de dados ocorreu entre janeiro de 2019 a março de 2020, por meio de entrevista contemplando um instrumento de avaliação do conhecimento sobre anticoagulante oral, cujo escore mínimo esperado é de zero e o máximo de 32 pontos, sendo que os maiores escores indicam um maior conhecimento. Foram excluídos os pacientes que não apresentaram condições cognitivas. Resultados: a maioria do sexo feminino vivia com companheiro, e estava com a situação profissional inativa, com média de idade de 49,9 anos. A média do escore do conhecimento geral foi de 19,8 pontos, média distante dos valores que indicam maior conhecimento. Já a média do escore do conhecimento específico foi de 3,0 pontos, o que demonstra maior conhecimento. Conclusão: observou-se déficit no conhecimento com relação ao exame de controle da coagulação e às condutas frente à interrupção do uso do anticoagulante, o que reforça a necessidade do acompanhamento criterioso e orientações ao paciente durante o tratamento.

Descritores: Enfermagem Perioperatória; Conhecimento; Anticoagulantes; Procedimentos Cirúrgicos Cardíacos; Implante de Prótese de Valva Cardíaca.

1 Enfermeira. Graduada. Escola de Enfermagem de Ribeirão Preto da Universidade de São Paulo. Egressa. Ribeirão Preto, São Paulo, Brasil. E-mail: carolina.galli.silveira@usp.br - ORCID iD: 0000-0002-9497-3481

2 Enfermeira. Mestre. Escola de Enfermagem de Ribeirão Preto da Universidade de São Paulo. Discente da Pósgraduação. Ribeirão Preto, São Paulo, Brasil. E-mail: paollaalgarte@yahoo.com.br - ORCID iD: 0000-0001-85834140

3 Enfermeira. Mestre. Escola de Enfermagem de Ribeirão Preto da Universidade de São Paulo. Discente da Pósgraduação. Ribeirão Preto, São Paulo, Brasil. E-mail: suellenromaier@usp.br - ORCID iD: 0000-0002-4677-1674

4 Enfermeira. Doutora. Escola de Enfermagem de Ribeirão Preto da Universidade de São Paulo. Professora Doutora. Ribeirão Preto, São Paulo, Brasil. E-mail: camarosti@usp.br - ORCID iD: 0000-0002-5521-8416

\footnotetext{
Autor Correspondente

Carina Aparecida Marosti Dessotte.

Endereço: Avenida dos Bandeirantes, 3900, Campus Universitário -

Bairro Monte Alegre. Ribeirão Preto, São Paulo, Brasil.

Telefone: (16) 3315-3410.

E-mail: camarosti@usp.br
}

Data de submissão: 01/04/2021

Data de aceite: 04/11/2021

\section{Como citar esse artigo:}

SILVEIRA, C.G; et al. Impacto da Intervenção Conhecimento de pacientes sobre anticoagulação oral após o implante de valva metálica. Advances in Nursing and Health, v. 4, p. 01-15, Londrina, 2022. 


\section{INTRODUÇÃO}

No Brasil, as valvopatias representam uma parcela significativa das internações por doenças cardiovasculares(1). Em grande parte dos casos, as internações revelam um comprometimento valvar acentuado, visto que as manifestações clínicas ocorrem tardiamente, em função do período assintomático característico no início da doença (2).

Atualmente, existem diversas formas de tratamento para as valvopatias, entre essas a correção cirúrgica (2). A partir da indicação de cirurgia de troca de valva cardíaca opta-se pela prótese biológica ou pela metálica. Não existe um substituto ideal para cada necessidade de substituição de prótese valvar, no entanto, existe um baixo risco de eventos tromboembólicos e hemorragias na utilização de prótese biológica, entretanto, a sua durabilidade é curta. Por outro lado, a prótese metálica possui maior durabilidade, porém com maior risco de eventos hemorrágicos ou tromboembólicos (3).

Os pacientes com próteses cardíacas metálicas necessitam da prevenção antitrombótica(4), com o uso de anticoagulantes orais (ACO), sendo os antagonistas da vitamina $\mathrm{K}$ os mais utilizados $^{(5)}$, todavia, possuem como principal complicação o sangramento, cujos determinantes são a intensidade, a duração da anticoagulação, o uso de medicamentos concomitantes, alimentos ricos em vitamina $\mathrm{K}$ e as características do paciente(6).

Os pacientes submetidos ao implante de prótese cardíaca valvar, na alta hospitalar, geralmente, são orientados a fazer uso contínuo do ACO antagonista da vitamina K. Dada a complexidade das reações adversas e à necessidade de verificar quão adequadas foram as orientações recebidas acerca da terapêutica medicamentosa após a alta se faz necessário o conhecimento sobre a ação e as complicações inerentes a essa terapia, por parte desses pacientes. Ademais, no cenário brasileiro, o processo de habilitação cardíaca, por meio do seguimento ambulatorial, está restrito a poucas instituições hospitalares.

Após revisão de literatura se verificou que são escassas as pesquisas desenvolvidas com o objetivo de avaliar o conhecimento desses pacientes sobre anticoagulação oral $(A C O)^{(7-8)}$ após a alta, entretanto, as avaliações ocorreram por meio de instrumentos submetidos apenas à validação 
de face. Os estudos(7-8) demonstraram conhecimento sobre a utilização do anticoagulante oral, no entanto, não trazem informações acerca da validade de estrutura interna dos instrumentos, o que impediu classificar o conhecimento quanto ao tipo adequado ou inadequado a partir dos escores. Todavia, os estudos(7-8) revelaram a necessidade de implementar o conhecimento dos pacientes acerca do exame de controle da anticoagulação, aspectos voltados à importância do uso ininterrupto da medicação e manutenção de uma dieta equilibrada, coerentemente com os efeitos do anticoagulante.

Frente aos achados, justifica-se esta investigação, visto que os pacientes submetidos ao implante de prótese valvar metálica necessitam de orientações acerca do medicamento em questão, quanto ao seu uso contínuo e às repercussões com relação ao controle da coagulação, dado este que pode favorecer a implementação da assistência de enfermagem no momento da alta e no seguimento ambulatorial. Diante do exposto, o presente estudo teve por objetivo avaliar o conhecimento sobre a anticoagulação oral de pacientes, após a primeira cirurgia cardíaca com implante de valva metálica.

\section{MÉTODO}

Trata-se de um estudo descritivo de corte transversal e abordagem quantitativa, realizado em um serviço de Terapia de Anticoagulação Oral (TACO), o qual realiza o seguimento ambulatorial dos pacientes (checagem dos valores do International Normalized Ratio - INR e agendamento de retorno), por meio de um protocolo institucional coordenado por enfermeiros e médicos de um hospital universitário localizado na cidade de Ribeirão Preto, em São Paulo (SP).

Não foi possível realizar um cálculo de tamanho amostral, pois não há disponível na literatura a variância das variáveis resposta e com a utilização do mesmo instrumento para revelar o construto em questão. Desse modo, uma amostra consecutiva e não probabilística foi constituída pelos pacientes que atenderam aos critérios de inclusão: ambos os sexos, com idade acima de 18 anos, independente da classe social e raça, que foram previamente submetidos à primeira cirurgia de correção de valvopatias, com implante de 
prótese metálica. Foram excluídos os pacientes que não apresentaram condições cognitivas. Para identificar os pacientes que possuem condições cognitivas para responder às perguntas subsequentes da entrevista, foram utilizadas seis questões: "Qual a data de hoje?", "Qual a sua idade?", "Em que dia da semana estamos?", "Qual o nome do local que estamos nesse momento?"; "Qual o seu nome completo?", e "Qual o nome da cidade em que você nasceu?". Os participantes foram excluídos do estudo ao errarem ou se não soubessem informar três ou mais questões $^{(9-10)}$.

A coleta de dados foi realizada na data do retorno ambulatorial para acompanhamento e controle da TACO, por meio de entrevistas individuais e consulta aos prontuários dos participantes utilizando instrumento contendo as variáveis sociodemográficas e clínicas: datas de internação, da entrevista e de nascimento; sexo; estado civil; escolaridade e situação profissional; renda mensal familiar; diagnóstico principal; comorbidades; hábitos de vida (tabagismo atual ou pregresso); data da cirurgia, cirurgia realizada; data do início da TACO e nome do ACO utilizado no pós-operatório mediato (PO). Foi perguntado ao paciente se ele recebeu orientação sobre a TACO, se sim, qual profissional the prestou a orientação?

Para a avaliação do conhecimento do paciente sobre a anticoagulação oral foi utilizado o "Instrumento de avaliação do conhecimento sobre anticoagulante oral (IACACO)", construído e validado por pesquisadores brasileiros(11). Trata-se de um instrumento composto por 32 questões, sendo dividido em dois módulos: o módulo geral para homens e mulheres; e o módulo específico apenas para mulheres em idade fértil. Nos dois módulos, cada questão correta equivale a um ponto e a incorreta não é atribuída pontuação, assim, o escore mínimo esperado é de zero e o máximo é de 32 pontos, com maiores escores indicando maior conhecimento sobre a TACO. O instrumento em questão possui evidências de validade de conteúdo, de construto e de confiabilidade. As autoras do instrumento não investigaram as evidências de validade de critério, o que não permite parametrizar os níveis de conhecimento. Os itens do IACACO foram corrigidos como certo ou errado, de acordo com o gabarito disponibilizado pelas autoras do 
instrumento(11).

Os dados foram inseridos no programa Office Excel 2010 com a técnica de dupla digitação das respostas obtidas e posterior verificação. Após a validação, os dados foram transportados para o Programa IBM-SPSS, versão 24.0 para Windows (SPSS, Inc., Chicago, IL, USA). Foram realizadas análises descritivas de frequência simples para variáveis nominais ou categóricas, de tendência central (média) e dispersão (desvio-padrão (DP) para as variáveis contínuas.

Os dados foram coletados após a aprovação pelo Comitê de Ética em Pesquisa da Escola de Enfermagem de Ribeirão Preto da Universidade de São Paulo, sob o número 2.972.451 e assinatura do Termo de Consentimento Livre e Esclarecido (TCLE) pelos participantes, que aceitaram participar do estudo.

\section{RESULTADOS}

Amostra constituída por 32 pacientes que estavam sendo acompanhados no ambulatório da referida instituição e a coleta de dados ocorreu entre janeiro de 2019 e março de 2020. A caracterização sociodemográfica da amostra se encontra na tabela 1 e na tabela 2 a caracterização clínica dos participantes.

A maior parte dos pacientes que realizou a cirurgia de troca valvar era do sexo feminino, vivia com companheiro e estava com a situação profissional inativa no momento da entrevista. Encontrou-se também uma baixa escolaridade e baixa renda mensal familiar. A média da idade dos pacientes, que foram entrevistados, era de 49,9 anos (DP=10,7).

Observa-se que na amostra a válvula mitral foi a mais acometida, seguida da aórtica. A doença valvar mais frequente foi a insuficiência valvar, seguida pela dupla lesão, insuficiência e estenose. Nota-se, ainda, que a maioria dos pacientes apresentava Hipertensão Arterial Sistêmica, e uma grande parte Fibrilação Atrial.

Em relação à cirurgia realizada e ao posicionamento de troca da valva, 17 pacientes $(53,1 \%)$ foram submetidos à troca da valva mitral, nove $(28,1 \%)$ a troca da valva aórtica e seis $(18,8 \%)$ a troca da valva mitral e aórtica.

Na tabela 3 se encontra a distribuição das frequências de acerto dos itens das 
respostas do instrumento de conhecimento

de ACO. A média apresentada sobre o tempo de uso de ACO foi de 44,78 meses $(D P=43,7)$ e mediana de 26 meses.

Tabela 1- Caracterização sociodemográfica dos participantes $(n=32)$. Ribeirão Preto, SP, Brasil 2019-2020

\begin{tabular}{lcc}
\hline Variáveis & Média (DP) & $\mathbf{n}(\%)$ \\
Sexo & $17(53,1)$ \\
Feminino & $15(46,9)$ \\
Masculino & \\
Vivendo com companheiro & $23(71,9)$ \\
Sim & $9(28,1)$ \\
Não & \\
Situação Profissional & \\
Inativo & $5(84,4)$ \\
Ativo & $49,6)$ \\
Idade & $6,4(10,7)$ & \\
Escolaridade (anos completos) & $6,5)$ \\
Renda Mensal (em reais) & $1480,3(831,3) * *$ & \\
\hline
\end{tabular}

Tabela 2 - Caracterização clínica dos participantes (n = 32). Ribeirão Preto, SP, Brasil 2019-2020.

\section{Variável}

\section{Válvula acometida}

Mitral

Aórtica

Mitral + Aórtica

Tricúspide

Mitral + Tricúspide

\section{Doença valvar}

Insuficiência

Insuficiência + Estenose

Estenose

Diagnóstico ignorado

\section{Comorbidades}

Hipertensão Arterial Sistêmica

Fibrilação Atrial

Diabetes Mellitus

Flutter

Dislipidemia

Insuficiência Cardíaca

\section{Tabagismo}

Pregresso

Atual

\section{n $(\%)$}

$16(50,0)$

$10(31,3)$

$4(12,5)$

$1(3,1)$

$1(3,1)$

$11(34,4)$

$10(31,2)$

$7(21,9)$

$4(12,5)$

$17(53,1)$

$12(37,5)$

$7(21,9)$

$7(21,9)$

$7(21,9)$

$6(18,8)$ 

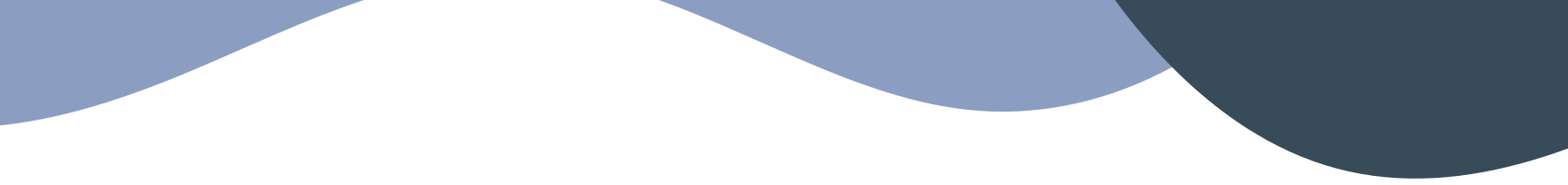

Tabela 3 - Distribuição das frequências de acerto dos itens das respostas do "Instrumento de avaliação do conhecimento sobre anticoagulante oral (IACACO)" dos participantes. Ribeirão Preto, SP, Brasil 2019-2020

Instrumento de avaliação do conhecimento sobre anticoagulante oral $(\mathbf{n}=32)$
Respostas

corretas n (\%)

13. Quais dos alimentos abaixo podem alterar o efeito do anticoagulante oral?

$31(96,9)$

1. Qual dos medicamentos abaixo é seu anticoagulante oral?

$30(93,8)$

2. No seu caso, qual foi a indicação para o uso do anticoagulante oral?

$30(93,8)$

16. Quais alimentos abaixo você foi orientado a evitar ou não consumir em excesso

$30(93,8)$ por causa do anticoagulante oral?

4. Por quanto tempo você irá usar o anticoagulante oral?

$29(90,6)$

5. Você sabe quando poderá interromper o uso do anticoagulante oral?

$29(90,6)$

$29(90,6)$

12. Enquanto estiver usando anticoagulante oral, quando você deverá realizar o exame de sangue (INR*)?

14. Antes de realizar uma viagem longa, o que você deve fazer?

$29(90,6)$

$26(81,3)$

$26(81,3)$

25. Em qual das situações abaixo é preciso informar que você faz uso de anticoagulante oral?

17. O INR* é um exame de sangue realizado para controlar a coagulação do sangue

$26(81,3)$ com o objetivo de:

15. Você conhece o motivo para o qual está usando o anticoagulante oral?

$26(81,3)$

$26(81,3)$

estiver usando anticoagulante oral?

9. Quais das situações abaixo podem ocorrer por complicação do uso do

$24(75,0)$ anticoagulante oral?

8. Se houver necessidade de você ser submetido a algum tipo de cirurgia ou tratamento dentário, o que você deve fazer?

7. Se o valor do seu INR* estiver acima do indicado para o seu problema de saúde, o que pode acontecer?

20. Se você usa anticoagulante oral e for consumir bebidas alcoólicas tais como pinga e outros destilados, cerveja, chopp ou vinho, quais os cuidados que deve ter?

28. Em qual das situações abaixo é necessário procurar, urgentemente, atendimento de saúde?

10. Ao perceber que se esqueceu de tomar a dose do anticoagulante oral do dia anterior, o que é correto fazer?

24. Caso você apresente sintomas de resfriado, gripe ou dor na garganta, o que poderá fazer enquanto estiver usando o anticoagulante oral?

22. Remédios caseiros, (chás de ervas e raízes), remédios fitoterápicos (ginckobiloba e cáscara sagrada) podem provocar o que nas pessoas que usam anticoagulante oral?

23. Dos cuidados abaixo descritos, quais os mais importantes para quem faz tratamento com anticoagulante oral? 
Continuação da tabela 3

19. Qual dos valores abaixo é o resultado ideal do INR* para o seu problema de

$17(53,1)$ saúde?

27. Antes de realizar uma viagem longa, o que você deve fazer?

$16(50,0)$

21. Quais das atividades abaixo é preciso evitar durante seu tratamento com $15(46,9)$ anticoagulante oral?

18. Qual é a vitamina que pode alterar na ação do anticoagulante oral?

$15(46,9)$

26. Caso você tenha deixado de tomar o anticoagulante oral por alguns dias (por exemplo, por 4 dias) o que é correto fazer?

3. Você sabe qual o nome do exame de sangue que é realizado para controlar a coagulação do sangue?

Específico para mulheres em idade fértil (que ainda menstruam) $(n=8)$

30. No caso de suspeita ou confirmação de gravidez durante o tratamento com anticoagulante oral o que você deve fazer?

29. Caso você queira engravidar durante o tratamento com anticoagulante oral, qual cuidado deve ter?

32. O uso do anticoagulante oral pode:

31. Quando uma mulher engravida durante o tratamento com anticoagulante oral o que pode ocorrer?

INR*: International Normalized Ratio

Em relação ao escore do

conhecimento geral sobre o uso do ACO, observou-se uma média de 19,8 ( $\mathrm{DP}=4,1)$ acertos, com mediana de 20,5. O intervalo obtido foi de 12 a 26. Quanto ao escore do conhecimento específico das mulheres, que ainda estão em idade fértil $(n=8)$, foi observada uma média de 3,0 (DP=1,0), com mediana $=3$, e o intervalo foi de 1 a 4 . As questões foram apresentadas na tabela 3, obedecendo a ordem sequencial proposta pelos autores, que validaram o instrumento.

A média dos valores dos escores apresentados sobre o conhecimento geral dos pacientes esteve próxima ao valor máximo a ser pontuado no instrumento, todavia, a mediana revelou a existência de escores muito inferiores que remetem ao déficit do conhecimento sobre o controle da coagulação e interrupção do tratamento com ACO. Sobre o conhecimento específico foram apresentados escores próximos ao valor máximo a ser pontuado no instrumento. 


\section{DISCUSSÃO}

Em resposta ao objetivo do estudo foi possível verificar que a média do escore do conhecimento geral foi de 19,8 pontos, já a média do escore do conhecimento específico foi de 3,0 pontos, no entanto, embora o escore médio esteja próximo da pontuação de melhor conhecimento, observou-se déficit em alguns itens avaliados pelo instrumento, o que reforça a necessidade de orientações na alta e acompanhamento ambulatorial criterioso aos pacientes submetidos a implante de prótese cardíaca valvar metálica.

Tais achados corroboram com os achados de estudos anteriores(7-8), que mensuraram o conhecimento sobre 0 anticoagulante oral, todavia, os aspectos relacionados ao construto conhecimento são distintos, pois no estudo em questão aspectos voltados ao controle do INR e ao uso contínuo do medicamento apresentaram escores, que demonstram conhecimento restrito dos pacientes investigados.

O perfil da amostra estudada corroborou com achados encontrados em outros estudos(12-13) sobre o conhecimento de pacientes a respeito da ACO, visto que houve o predomínio de pacientes do sexo feminino, casados, inativos profissionalmente, com baixa escolaridade e renda mensal.

A média de idade encontrada, em um estudo brasileiro(12), no qual os pesquisadores avaliaram 110 pacientes portadores de prótese cardíaca valvar metálica, em relação ao conhecimento sobre o uso da ACO, foi de 50,3 anos, semelhante com a média de idade da presente investigação, possivelmente, em função da opção pela prótese metálica ser comum em pacientes adultos em decorrência de sua durabilidade.

Entre os fatores sociais, a baixa escolaridade pode interferir na compreensão dos participantes quanto à terapia utilizada, durante a orientação na alta e/ou durante o acompanhamento ambulatorial. Com relação à baixa renda, essa pode interferir no que diz respeito à aquisição de medicamentos e a realização periódica de exames, o que pode levar a um controle inadequado do INR durante o tratamento(13-14).

O predomínio de acometimento da valva mitral, seguido pelo comprometimento 
da valva aórtica, também foi encontrado em um estudo que verificava o conhecimento sobre a $\operatorname{TACO}(7,14)$. Com relação às comorbidades apresentadas, a hipertensão arterial sistêmica (HAS) e a fibrilação atrial (FA) foram as mais prevalentes entre a amostra estudada, seguidas pelas dislipidemias e diabetes mellitus, dados que corroboram com um estudo realizado em um Estado do nordeste brasileiro (12).

Em relação aos hábitos de saúde, o tabagismo, ativo ou pregresso, pode ter comprometido a evolução da doença valvar e, ainda, pode comprometer o tratamento. É imperativo afirmar, que o conhecimento satisfatório sobre anticoagulação oral fortalece a inserção da utilização do medicamento nas atividades de vida diária dos valvopatas(15).

Os ACO são, usualmente, indicados para indivíduos com doenças cardiovasculares, com risco para reações tromboembólicas(16-17). Um estudo alemão, com pacientes após o implante valvar, reforçou a importância de os pacientes serem informados sobre os cuidados após o implante de valva cardíaca metálica, com ênfase ao uso contínuo de $\mathrm{ACO}\left({ }^{18)}\right.$.

O conhecimento sobre a posologia do ACO pode ser considerado um fator determinante para a utilização coerente com a necessidade de saúde do indivíduo após o implante de valva cardíaca metálica. O ACO possui dosagem inicial variando entre 2,5 a $5 \mathrm{mg} / \mathrm{dia}$, necessitando de controle laboratorial do INR, devendo este ser realizado após o quinto dia da intervenção cirúrgica, e logo após os ajustes deve estabelecer a dose adequada para cada caso específico $^{(6,17)}$.

A monitorização laboratorial deve ser realizada pelo INR, exame este calculado a partir das atividades de protrombina, refletindo o tempo de coagulação sanguínea. O acompanhamento do INR durante o tratamento com ACO é importante, pois possibilita o ajuste de dose e previne os eventos adversos da medicação(6,19-20). É importante que o paciente compreenda como deve ocorrer a monitorização do INR, para isso, é de suma importância que este receba as devidas orientações na alta, após o procedimento cirúrgico, a fim de evitar eventos adversos em decorrência do uso do ACO.

Compreender os riscos de complicações hemorrágicas e tromboembólicas inerentes à terapia é 


\section{He}

importante para o reconhecimento de sinais e sintomas, que denotem a necessidade urgente de avaliação médica(20), para isso as orientações na alta e o reforço de tais informações no acompanhamento ambulatorial são imprescindíveis.

Os analgésicos e os antipiréticos podem potencializar os efeitos ACO, pois possuem ação que impede a agregação plaquetária. O uso de chás e fitoterápicos precisam ser monitorizados, visto que podem provocar a alteração na coagulação sanguínea(19). Em decorrência destes eventos, o paciente deve ser informado sobre essas particularidades do uso de ACO, para que entenda todo 0 processo terapêutico.

As interações medicamentosas entre - ACO e outros medicamentos podem provocar alteração do INR. A amiodarona ${ }^{\circledR} \mathrm{e}$ a sinvastatina ${ }^{\circledR}$ interferiram diretamente na dose terapêutica do ACO, a amiodarona ${ }^{\circledR}$ em função da redução do metabolismo hepático e a sinvastatina ${ }^{\circledR}$ por se ligar às proteínas plasmáticas, potencializando o efeito do ACO e favorecendo o risco de sangramento(21).

O hábito alimentar possui relação direta com os valores apresentados no INR, pois os alimentos ricos em vitamina $K$, quando ingeridos em grande quantidade, potencializam o efeito anticoagulante. Já a diminuição destes alimentos pode aumentar o risco de formação de trombos. Desse modo, o paciente precisa ser orientado sobre quais alimentos são ricos em vitamina $K^{(21)}$.

$$
\text { É necessário que o paciente }
$$
compreenda que o uso esporádico de bebidas alcoólicas promoverá a redução do metabolismo do ACO, potencializando o seu efeito e elevando o risco de sangramento. Por outro lado, o uso contínuo de bebidas alcoólicas aumentará o metabolismo do ACO, reduzindo o seu efeito farmacológico(22).

É sabido que o sedentarismo pode comprometer a manutenção dos valores terapêuticos do INR e a realização regular de atividade física pode minimizar os riscos de complicações relacionadas ao uso do $\mathrm{ACO}(21)$. Entretanto, toda e qualquer atividade, seja esportiva ou doméstica, em que haja a possibilidade acidental de sangramento, deve ser evitada durante a terapia.

A falta de conhecimento do paciente sobre o uso diário do medicamento pode prejudicar a eficácia da TACO, uma vez que potencializa os riscos de complicações geradas pela terapêutica medicamentosa 
descontinuada na ocorrência de viagem $(16,21)$

O diálogo entre paciente e profissional de saúde se torna primordial para a eficácia das ações voltadas à promoção, à prevenção e à reabilitação da saúde do indivíduo em uso de ACO. Informar ao profissional de saúde sobre o uso contínuo do medicamento é preponderante para a tomada de decisão direcionada à manutenção da adesão ao tratamento dentro da faixa terapêutica (21).

Em mulheres que possuam prótese valvar metálica, o uso de ACO contribuirá para o aumento do fluxo e/ou o aumento do período menstrual. A presença de prótese valvar metálica, em mulheres grávidas, possui um elevado risco de complicações tromboembólicas, sendo de suma importância a manutenção da anticoagulação durante o período gravídico e puerpério (23).

Frente aos achados relacionados ao conhecimento dos participantes sobre o uso do ACO se observou carência de estudos, que evidenciassem 0 conhecimento do respectivo público, o que limitou confrontar os dados obtidos neste estudo com outras investigações. Outra limitação do estudo foi o número restrito de participantes, tendo em vista que a coleta de dados precisou ser interrompida diante da pandemia que se instalou no Brasil e no mundo, no começo do ano 2020. O cenário pandêmico atual reforça a necessidade de orientações eficientes sobre a TACO na alta e durante o acompanhamento ambulatorial dos valvopatas, após o implante de prótese valvar metálica.

\section{CONCLUSÃO}

Em síntese, é possível concluir que os resultados apontam para a existência de conhecimento sobre o uso de anticoagulante oral por parte dos pacientes, após a primeira cirurgia cardíaca, com implante de valva metálica, todavia, aspectos voltados ao fortalecimento sobre a importância do uso contínuo da medicação ainda são importantes para uma melhor qualidade de vida durante a terapia medicamentosa. 


\section{REFERÊNCIAS}

1. Tarasoutchi F, Montera MW, Ramos AIO, Sampaio RO, Rosa VEE, Accorsi TAD et al. Atualização das Diretrizes Brasileiras de Valvopatias - 2020. Arq Bras Cardiol. v. 115, n. 4, p. 720-775, Oct. 2020. Disponível em: <http://www.scielo.br/s cielo.php? script $=$ s ci_ artt ext\&pid $=$ S0066$782 \times 2020001200720 \& \mid n g=e n \& n r m=i s o>$. access on 10 May 2021. Epub Oct 23, 2020. https://doi.org/10.36660/abc.20201047.

2. Ramos AIO, Accorsi TAD, Jerônimo AD. Insuficiência mitral. In: Magalhães CC. et al. Tratado de cardiologia SOCESP. Barueri: Manole; 2015. p. 791-796.

3. Fernandes AMS, Pereira FS, Bitencourt LS, Pereira NAV, Bastos GB, Durães AR et al. A influência da escolha da prótese valvar sobre a mortalidade intra-hospitalar no pósoperatório em pacientes submetidos à cirurgia valvar. Rev Bras Cir Cardiovasc. 2014;29(4):559-63. Disponível em: https://www.scielo .br/ sc ielo.php?script=sci_abstract\&pid $=$ S0102$76382014000400013 \&$ Ing $=e s \& n r m=i s o \& t \ln$ $\mathrm{g}=\mathrm{pt}$

4. Lorga FAM, Azmus AD, Soeiro AM, Quadros AS, Avezum JA, Marques AC et al. Diretrizes brasileiras de antiagregantes plaquetários e anticoagulantes em cardiologia. Arq Bras Cardiol. 2013;101 (suppl 3:S01-95). Disponível em: http://publicacoes.cardiol.br/consenso/2013/ Diretriz_Antiagregantes_Anticoagulantes.asp

5. Valvulopathies ATI. Terapia anticoagulante nas valvopatias. Rev Soc Cardiol Estado de São Paulo. 2017; 27(3): 228-33.

6. Terra-Filho M, Menna-Barreto SS. Recomendações para o manejo da tromboembolia pulmonar, 2010. J bras pneumol. 2010;36(Suppl1):S01-68. Disponível em: https://www.scielo.br/pdf/jbpneu/v36s1/v36 s1a01.pdf

7. Rocha HT, Rabelo E, Aliti G, Souza EN. Knowledge of Patients with Mechanical Valve Prostheses Concerning Chronic Oral Anticoagulant Therapy. Rev Latino-Am Enfermagem. 2010;18(4):S696-702. Disponível em: https://www.scielo.br/ scielo.php?scri pt=sci_arttext\& pid=S010411692010000400006

8. Cao H, Wu T, Chen W, Fu J, Xia X, Zhang J. The effect of warfarin knowledge on anticoagulation control among patients with heart valve replacement. Int J Clin Pharm. 2020;42(3):861-70.

DOI: https://doi.org/10.1007/s11096-020-01043y

9. Pfeiffer E. A Short Portable Mental Status Questionnaire for the Assessment of Organic Brain Deficit in Elderly Patients. Journal of the American Geriatrics Society. 1975; 23(10):433-41. Disponível em: 
https://onlinelibrary.wiley.com/doi/epdf/10.1 111/j.1532-5415.1975.tb00927.x

10. Silva LN. Comparação de três instrumentos para avaliação da fadiga em pacientes com insuficiência cardíaca. Universidade de São Paulo [Dissertação] 2016. [cited 2018 jun 1]. Disponível em: https://www.teses. usp.br/teses\%20/disponiveis/22/22132/tde26012017-103129/publico/\%20LUMA\% 20NASCIMEM TOSILVA.pdf

11. Garcia FSS, Cunha DPT, Manzato RO, Bolela F, Barbetta PA, Dantas RS. Assessing Knowledge of Oral Anticoagulant Therapy: Construction and Validation. Journal of Nursing Meas. 2018;26(1): S53-73. Disponível em: https://pubmed.ncbi.nlm.nih.gov/29724279/

12. Parys, M, Kowalczuk-Wieteska A, Kulik H, Majchrzyk I, Zembala M. A novel survey examining the level of knowledge about anticoagulant and anti-infectious prophylaxis in patients after mechanical cardiac valve implantation. Kardiologia Polska (Polish Heart Journal). 2019; 77(2):225-227.

13. Figueirêdo TR, Nascimento MO, Silveira MMBM, Costa CRB, Queiroga AV, Bezerra SMMSB. Conhecimento de pacientes em acompanhamento ambulatorial sobre a terapia de anticoagulação oral. Rev pesqui cuid Fundam. 2016;8(1):3883-92. Disponível em:

http://www.seer.unirio.br/index.php\%20/cui dado\%20fundamental/article/view/5162/pd f_1806
14. Kakkar N, Kaur R, John M. Outpatient oral anti-coagulation management: an audit of 82 patients. J Assoc Physicians India. 2005;52:868-72. Disponível em: https://pubmed.ncbi.nlm.nih.gov/16459526/

15. Braz NJ. Fatores determinantes da infecção do sítio cirúrgico em pacientes submetidos a cirurgias de revascularização do miocárdio e implantes de válvulas cardíacas. Universidade Federal de Minas Gerais [Tese] 2017 [cited 2020 mai 12]. Disponível em: https://repositorio.ufmg.br/bitstream/1843/A NDO-AMUNG8/1 /dissert acao_de_mestra do_nelma_de_jesus_braz_2017.pdf

16. World Health Organization. The economics of tobacco and tobacco control. Geneva (Suiça): World Health Organization; 2016. Disponível em: https://cancercontrol .cancer.gov /brp/tcrb/monographs\%20/21/docs/m21_co mplete\%20.pdf

17. Serra IC, Ribeiro LCAN, Gemito MLGP, Mendes FRP. Manejo terapéutico de los usuarios con terapia anticoagulante oral. Enferm glob. 2016;15(41):S10-19. Disponível em: http://scielo.isciii.es/scielo.php?script=sci_ar ttext\&pid=S1695-61412016000100002\&Ing $=\mathrm{pt}$

18. Harter $\mathrm{K}$, Levine $\mathrm{M}$, Henderson SO. Anticoagulation Drug Therapy: A Review. West J Emerg Med. 2015;16(1):S11-17. Disponível em: 
https://www.ncbi.nlm.nih.gov/\%2 0pmc/ \%20ar \%20ticles /PMC4307693/pdf/wjem16-11.pdf

19. Kottmaier M, Hettich I, Deutsch M, Badiu C, Krane M, Ruediger L et al. Quality of Life and Anxiety in Younger Patients after Biological versus Mechanical Aortic Valve Replacement. Thorac Cardiovasc Surg. 2017; 65(3): 198-205.

DOI:

http//dx.doi.org/10.1055/s0036-1584907

20. Molina FT, Zanusso GJ. Anticoagulantes cumarínicos: ações, riscos e monitoramento da terapêutica. Saúde e Biologia. 2014;9(2):S75-82. Disponível em: http://revista2.grupointegrado.br/revista /index.php/sabios2/article/view/1263

21. Leal PM, Amante LN, Girondi JBR, Nascimento ERP, Magalhães ALP. Building solutions for the safety of the patient with heart disease using warfarin: a qualitative study. Texto contexto enferm. 2020;29:e20180002. DOI: https://doi.org/10.1590/1980-265x-tce2018-0002
22. Simonetti, SH; Mancussi, AC; Bianchi, F. Preditores clínicos intervenientes na adesão de usuários de anticoagulante orais. Enferm Foco. 2019;10:S02-06. Disponível em: http://ver ista .cofen.gov .br/index .php/ enfermagem/article/view/1359

23. Simonetti SH, Faro ACM, Bianchi ERF. Escore de Adesão para Usuários de Anticoagulantes Orais. Int J Cardiovasc Sci. 2018;31(4):S383-392.

DOI:

https://doi.org/10.5935/2359-

4802.20180028

24. Cavalcante Júnior JL, Nobre MN, Sena RG, Cavalcante JWS, Magalhães RT, Luna FMS. Anticoagulação em gestantes com prótese valvar mecânica. Rev Eletro Acervo da Saúde. 2019;20:S01-06. DOI: https://doi.org/10.25248/reas.e446.2019 